\title{
Determining dislocation Love numbers using satellite gravity mission observations
}

\author{
Wenke Sun, Shuhei Okubo, and Takayuki Sugano \\ Earthquake Research Institute, University of Tokyo, Yayoi 1-1-1, Bunkyo-ku, Tokyo 113-0032, Japan \\ (Received May 26, 2005; Revised January 10, 2006; Accepted January 13, 2006; Online published May 12, 2006)
}

\begin{abstract}
This paper presents a new approach to calculate dislocation Love numbers using observations of a satellite gravity mission (e.g. GRACE). The necessary condition is that the co-seismic potential change be sufficiently large to be detected by the gravity mission. Co-seismic deformations for each spherical harmonic degree $n$ are decoupled. Therefore, dislocation Love numbers of degree $n$ can be determined independently. The determinable maximum harmonic degree $n$ depends on the seismic size, source type, magnitude, and the accuracy of a satellite gravity mission. For an arbitrary seismic source, all four types of dislocation Love numbers can be determined using data from only one seismic event because all deformation components are involved together. Only the concerned dislocation Love numbers can be computed for any one of the four types of sources. To prove the validity of the method proposed in this study, a simulation test is carried out by considering a similar case to the 2004 Sumatra earthquake (Mw 9.1). Results show that the method works well and guarantee the accuracy.

Key words: Co-seismic deformation, dislocation Love number, gravity mission, earthquake GRACE, Sumatra earthquake.
\end{abstract}

\section{Introduction}

Dedicated satellite missions, such as The Gravity Recovery and Climate Experiment (GRACE) (NRC, 1997), are now available for gravity field determination from space. They provide an extremely accurate, global, and highresolution estimate of constant and time-variable components of the earth's gravity field every 30 days over a 5-year period (Wahr et al., 1998). It is anticipated that the gravity missions will yield extremely wide geophysical applications in geosciences, with measurement of temporal gravity variations caused by various geophysical processes including atmospheric mass redistribution, ocean circulation, polar ice melting or aggregation, visco-elastic response of the Earth's lithosphere to past and present loads, and others (Chao et al., 2000; Chao, 2003). In addition to these processes, earthquakes can produce large global gravity perturbations that are detectable through analysis of gravity mission data. Gross and Chao (2001) investigated gravity perturbations using normal mode technique based on Chao and Gross (1987). Comparing the degree amplitude spectra of some earthquakes with expected GRACE sensitivity, they concluded that co-seismic effects of great earthquakes such as the 1960 Chilean or 1964 Alaska events can cause global gravitational field changes that are sufficiently large as to be detectable by GRACE. Sun and Okubo (2004a, b) approached this problem from two perspectives. They derived theoretical formulations of co-seismic geoid and gravity changes and their degree variances, expressed by dislocation Love numbers. These expressions are achieved using

Copyright (c) The Society of Geomagnetism and Earth, Planetary and Space Sciences (SGEPSS); The Seismological Society of Japan; The Volcanological Society of Japan; The Geodetic Society of Japan; The Japanese Society for Planetary Sciences; TERRAPUB. the dislocation theory of Sun and Okubo (1993), for a spherical earth as it is expressed in the form of spherical harmonics. They investigated co-seismic geoid and gravity changes by observing the distribution of their degree variances in comparison to the expected sensitivity of satellite gravity missions. Co-seismic deformations for large earthquakes are discussed with respect to their detectability. Results indicate that both the gravity and geoid changes are near two orders of magnitude larger than the precision of gravity missions in low harmonic degrees. Based on those results, they derived the minimum magnitudes of earthquakes detectable by GRACE. Their conclusion was that co-seismic deformations for an earthquake with a seismic magnitude greater than $m=7.5$ (for tensile sources) and $m=9.0$ (for shear sources) are expected to be detectable by GRACE. Note that the dislocation Love numbers used in Sun and Okubo (2004a, b) are obtained conventionally for a spherically symmetric earth model such as the 1066A (Gilbert and Dziewonski, 1975) or the preliminary reference earth model (PREM) (Dziewonski and Anderson, 1981). However, dislocation Love numbers calculated based on an earth model are theoretically different from those of the earth. On the other hand, Okubo et al. (2002) showed that the co-seismic deformations vary if the earth parameters are adjusted. That fact implies that the accuracy of the dislocation Love numbers depends directly on the rightness of the adopted earth model. If possible, it is better to determine them by real observations because they carry real information regarding the earth's structure. Satellite gravity missions provide the possibility of determining the dislocation Love numbers.

Therefore, dislocation Love numbers are considered in this study as unknown variables. They are derived from observations of satellite gravity missions such as GRACE. 
Then theoretical formulations are presented for determining the dislocation Love numbers. Numerical applications should be useful for a seismic event that is sufficiently large to be detectable from space.

\section{Co-Seismic Potential Change and Dislocation Love Numbers}

Assume that an inclined point dislocation is located on the polar axis in a compressible and self-gravitating spherical earth, and that the fault line is in the direction of $\varphi=0$ (Greenwich meridian). According to the quasi-static dislocation theory, the co-seismic potential change at an observation point $(a, \theta, \varphi)$ can be expressed as (Sun and Okubo, 1993)

$$
\psi^{i j}(a, \theta, \varphi)=\sum_{n, m} k_{n m}^{i j} Y_{n}^{m}(\theta, \varphi) \cdot v_{i} n_{j} \frac{g_{0} U d S}{a^{2}},
$$

where $k_{n m}^{i j}$ (related to the gravitational potential change) are the dislocation Love numbers, function of the spherical harmonic degree, order, source depth, and source type. Components of the slip vector and its normal on the infinitesimal fault area $d S$ are $v_{i}$ and $n_{j}$ with total dislocation $U$. Gravity on the earth surface is $g_{0}, a$ is the Earth's radius, and $Y_{n}^{m}(\theta, \varphi)$ is the spherical harmonic function of degree $n$ and order $m$. The so-called dislocation factor, $g_{0} U d S / a^{2}$, defines the earthquake magnitude and gives the unit of potential change.

A combination of the three slip and three normal components means that nine solutions exist for all possible sources. However, only four independent solutions exist if the earth model is spherically symmetric and isotropic. A deformation caused by an arbitrary source can be obtained by a proper combination of the four types of independent sources. In this study, we choose the following four independent solutions: $i j=12,32,22$, and 33. They represent strike-slip, dip-slip, horizontal tensile and vertical tensile, respectively. Components of $i j=22$ include two parts: $m=0$ and 2. Computation of $m=2$ is derived easily from the component of $i j=12$. Expressions of the four independent solutions are given as the following (Sun and Okubo, 1993).

$$
\begin{aligned}
\psi^{12}(a, \theta, \varphi) & =2 \sum_{n=2}^{\infty} k_{n 2}^{12} P_{n}^{2}(\cos \theta) \sin 2 \varphi \frac{g_{0} U d S}{a^{2}} \\
\psi^{32}(a, \theta, \varphi) & =2 \sum_{n=1}^{\infty} k_{n 1}^{32} P_{n}^{1}(\cos \theta) \sin \varphi \frac{g_{0} U d S}{a^{2}} \\
\psi^{22}(a, \theta, \varphi) & =\left[\sum_{n=0}^{\infty} k_{n 0}^{22} P_{n}(\cos \theta)\right. \\
& \left.-2 \sum_{n=2}^{\infty} k_{n 2}^{12} P_{n}^{2}(\cos \theta) \cos 2 \varphi\right] \frac{g_{0} U d S}{a^{2}} \\
\psi^{33}(a, \theta, \varphi) & =\sum_{n=0}^{\infty} k_{n 0}^{33} P_{n}(\cos \theta) \frac{g_{0} U d S}{a^{2}}
\end{aligned}
$$

A co-seismic potential change caused by an arbitrary inclined fault can be expressed by the above four independent solutions. In this case, a dislocation vector $\boldsymbol{v}$ and its normal $\mathbf{n}$ can be described in terms of dip-angle $\delta$ and slip-angle $\lambda$ of the fault as

$$
\begin{aligned}
& \mathbf{n}=\mathbf{e}_{3} \cos \delta-\mathbf{e}_{2} \sin \delta \\
& \boldsymbol{v}=\mathbf{e}_{3} \sin \delta \sin \lambda+\mathbf{e}_{1} \cos \lambda+\mathbf{e}_{2} \cos \delta \sin \lambda .
\end{aligned}
$$

We face a shear dislocation problem if dislocation vector $\boldsymbol{v}$ runs parallel to the fault plane. Similarly, for a tensile opening, the dislocation vector $\boldsymbol{v}$ and its normal $\mathbf{n}$ become equal:

$$
\boldsymbol{v}=\mathbf{n}=\mathbf{e}_{3} \cos \delta-\mathbf{e}_{2} \sin \delta .
$$

Then for an arbitrary shear fault on the polar axis, according to Eqs. (1)-(5) the co-seismic potential change can be written as the following.

$$
\begin{aligned}
\psi^{\text {Shear }}(a, \theta, \varphi)= & \sum_{n=2}^{\infty}\{(\sin \lambda \sin 2 \delta \cos 2 \varphi \\
& -2 \cos \lambda \sin \delta \sin 2 \varphi) P_{n}^{2}(\cos \theta) k_{n 2}^{12} \\
& +2(\sin \lambda \cos 2 \delta \sin \varphi \\
& +\cos \lambda \cos \delta \cos \varphi) P_{n}^{1}(\cos \theta) k_{n 1}^{32} \\
& \left.+\frac{1}{2} \sin \lambda \sin 2 \delta P_{n}(\cos \theta)\left(k_{n 0}^{33}-k_{n 0}^{22}\right)\right\} \\
& \cdot \frac{g_{0} U d S}{a^{2}}
\end{aligned}
$$

Similarly, for a tensile source, the co-seismic potential change becomes the following.

$$
\begin{aligned}
\psi^{\text {Tensile }}(a, \theta, \varphi)= & \sum_{n=2}^{\infty}\left[\cos ^{2} \delta P_{n}(\cos \theta) k_{n 0}^{33}\right. \\
& +\sin ^{2} \delta P_{n}(\cos \theta) k_{n 0}^{22} \\
& -2 \sin ^{2} \delta \cos 2 \varphi P_{n}^{2}(\cos \theta) k_{n 2}^{12} \\
& \left.-2 \sin 2 \delta \sin \varphi P_{n}^{1}(\cos \theta) k_{n 1}^{32}\right] \\
& . \frac{g_{0} U d S}{a^{2}} .
\end{aligned}
$$

Dislocation Love numbers $k_{n m}^{i j}$ in (9) and (10) are obtained conventionally for a spherically symmetric earth model (Sun and Okubo, 1993) such as the 1066A (Gilbert and Dziewonski, 1975) or the preliminary reference earth model (PREM) (Dziewonski and Anderson, 1981). Once a dislocation source or earthquake parameter is provided, coseismic deformations can be calculated easily using these Love numbers. Subsequently, the potential change can be calculated by the above summations in (9) or (10). Note that the dislocation Love numbers $k_{n 0}^{33}-k_{n 0}^{22}$ in (9) can be considered as a whole since they appear in the formula in form of difference of the two tensile sources.

However, the dislocation Love numbers calculated based on an earth model are theoretically worse than those of the earth. Furthermore, Okubo et al. (2002) showed the dependence of co-seismic deformations on the earth parameters. That fact implies that the accuracy of the dislocation Love numbers depends directly on the adopted earth model. If possible, it is better to determine those using real observations. Satellite gravity missions provide just such a possibility. For that reason, dislocation Love numbers are considered to be unknown variables in this study, and are derived from observations of satellite gravity missions, such 
as GRACE. In previous studies, Sun and Okubo (2004a, b) proved that the satellite gravity mission (GRACE) is able to detect co-seismic deformations from space. This benefit allows the study of the earth's inner structure from a new vantage because dislocation Love numbers, when they are derived independently from space, carry reliable information of the earth's mass distribution. Therefore, the observed dislocation Love numbers are useful not only for geodetic application, but also for modeling the earth structure as a new condition, in combination with seismic knowledge.

Co-seismic deformations can be studied for individual harmonic degrees because the satellite gravity missions provide potential measurements in the form of spherical harmonic coefficients, as indicated by Chao and Gross (1987). Note that the terms of degrees $n=0$ and $n=1$ in Eqs. (9) and (10) vanish because the total mass of the earth is constant and the reference frame origin is located at the center of mass of the earth model. On the other hand, the angular order $m$ vanishes except $m=0,1$ and 2 because the source is chosen at the polar axis and because of the symmetric property of the source functions.

\section{Potential Change Observed by Satellite Gravity Missions}

On the other hand, satellite gravity missions provide the following observations for a potential anomaly as a spherical harmonic series (Heiskanen and Moritz, 1967):

$$
\begin{aligned}
T(a, \theta, \varphi)= & a \sum_{n=0}^{\infty} \sum_{m=-n}^{n}\left(\Delta C_{n m} \cos m \varphi\right. \\
& \left.+\Delta S_{n m} \sin m \varphi\right) P_{n}^{m}(\cos \theta)
\end{aligned}
$$

where $\Delta C_{n m}$ and $\Delta S_{n m}$ are differences of two sets of spherical harmonic coefficients $\left(C_{n m}^{1}, S_{n m}^{1}\right)$ and $\left(C_{n m}^{2}, S_{n m}^{1}\right)$ of the geo-potential model observed by the GRACE mission:

$$
\begin{aligned}
& \Delta C_{n m}=C_{n m}^{2}-C_{n m}^{1}, \quad \text { and } \\
& \Delta S_{n m}=S_{n m}^{2}-S_{n m}^{1} .
\end{aligned}
$$

Notice that a dislocation may occur at an arbitrary position in the earth in practice, whereas satellite gravity missions always provide results in spherical coordinates with the North Pole as orientation. Then the theoretical co-seismic potential changes expressed in (9) and (10) and the potential change provided by the satellite in (11) are for two different spherical coordinate systems: $(a, \theta, \varphi)$ and $\left(a, \theta^{\prime}, \varphi^{\prime}\right)$. For comparison, they must be unified into one coordinate system by transforming one of them to the other's format. Because of the spherical symmetric property, results are identical whichever is transformed. Nevertheless, the dislocation Love numbers in (9) and (10) are unknown and are derived from satellite observations. For that reason, it is convenient to leave them unchanged. On the other hand, if the seismic source is chosen at a pole, the co-seismic potential change only contains spherical harmonic orders $m=0$, 1 and 2. Otherwise, all spherical harmonic orders $m$ will be involved. Therefore, we transform Eq. (11) into the same system as that used in (9) and (10) below:

$$
T(a, \theta, \varphi)=a \sum_{n=0}^{\infty} \sum_{k=-n}^{n}\left(\Delta c_{n k} \cos k \varphi\right.
$$

$$
\left.+\Delta c_{n k} \sin k \varphi\right) P_{n}^{k}(\cos \theta)
$$

where

$$
\begin{aligned}
\Delta c_{n k} & =\sum_{m=0}^{n} a_{n m}^{k} \Delta C_{n m} \\
\Delta s_{n k} & =\sum_{m=0}^{n} b_{n m}^{k} \Delta S_{n m}
\end{aligned}
$$

and coefficients $a_{n m}^{k}$ and $b_{n m}^{k}$ can be obtained by a set of reoccurrence formulas, as listed in Appendix A.

\section{Dislocation Love Numbers Derived From Ob- servations of Gravity Missions}

Theoretically, the predicted potential change $\psi^{\text {Shear }}(a, \theta, \varphi) \quad\left(\right.$ or $\left.\quad \psi^{\text {Tensile }}(a, \theta, \varphi)\right)$ should be identical to the observed $T(a, \theta, \varphi)$ anywhere on the earth surface, as

$$
\psi(a, \theta, \varphi) \equiv T(a, \theta, \varphi) .
$$

In practice, the use of either $\psi^{\text {Shear }}(a, \theta, \varphi)$ or $\psi^{\text {Tensile }}(a, \theta, \varphi)$ depends on the actual source typeshear or tensile. On the other hand, relation (17) holds for any harmonic degree. Consequently, we only discuss spherical harmonic degree $n$ in the following. Assuming a shear seismic source, inserting (9) and (14) into (17) yields

$$
\begin{aligned}
& f_{1}(\theta, \varphi) k_{n 2}^{12}+f_{2}(\theta, \varphi) k_{n 1}^{32}+f_{3}(\theta, \varphi)\left(k_{n 0}^{33}-k_{n 0}^{22}\right) \\
& \quad=g(\theta, \varphi)
\end{aligned}
$$

where

$$
\begin{aligned}
f_{1}(\theta, \varphi)= & (\sin \lambda \sin 2 \delta \cos 2 \varphi \\
& -2 \cos \lambda \sin \delta \sin 2 \varphi) P_{n}^{2}(\cos \theta) \frac{g_{0} U d S}{a^{2}} \\
f_{2}(\theta, \varphi)= & 2(\sin \lambda \cos 2 \delta \sin \varphi \\
& +\cos \lambda \cos \delta \cos \varphi) P_{n}^{1}(\cos \theta) \frac{g_{0} U d S}{a^{2}} \\
f_{3}(\theta, \varphi)= & \frac{1}{2} \sin \lambda \sin 2 \delta P_{n}(\cos \theta) \frac{g_{0} U d S}{a^{2}} \\
g(\theta, \varphi)= & a \sum_{k=-n}^{n}\left(\Delta c_{n k} \cos k \varphi\right. \\
& \left.+\Delta c_{n k} \sin k \varphi\right) P_{n}^{k}(\cos \theta) .
\end{aligned}
$$

Note that $f_{i}(\theta, \varphi)(i=1,2,3)$ and $g(\theta, \varphi)$ in (18) are known once the parameters of an earthquake are provided. The only unknowns are the dislocation Love numbers $k_{n 2}^{12}$, $k_{n 1}^{32}$ and $k_{n 0}^{33}-k_{n 0}^{22}$. The remaining task is to solve Eq. (18). For that purpose, (18) can be discretized into a linear system

$$
\mathbf{F K}=\mathbf{G},
$$

where

$$
\begin{aligned}
\mathbf{K} & =\left(k_{n 2}^{12}, k_{n 1}^{32}, k_{n 0}^{33}-k_{n 0}^{22}\right)^{\mathrm{T}} \\
\mathbf{G} & =\left(g\left(\theta_{1}, \varphi_{1}\right), g\left(\theta_{2}, \varphi_{2}\right), \cdots g\left(\theta_{N}, \varphi_{N}\right)\right)^{\mathrm{T}} \\
\mathbf{F} & =\left(\begin{array}{ccc}
f_{1}\left(\theta_{1}, \varphi_{1}\right) & f_{2}\left(\theta_{1}, \varphi_{1}\right) & f_{3}\left(\theta_{1}, \varphi_{1}\right) \\
f_{1}\left(\theta_{2}, \varphi_{2}\right) & f_{2}\left(\theta_{2}, \varphi_{2}\right) & f_{3}\left(\theta_{2}, \varphi_{2}\right) \\
\ldots & \cdots & \cdots \\
f_{1}\left(\theta_{N}, \varphi_{N}\right) & f_{2}\left(\theta_{N}, \varphi_{N}\right) & f_{3}\left(\theta_{N}, \varphi_{N}\right)
\end{array}\right)
\end{aligned}
$$


So that the unknown dislocation Love numbers $\mathbf{K}$ can be obtained easily as

$$
\mathbf{K}=\mathbf{F}^{-1} \mathbf{G} .
$$

Decoupling of the dislocation Love numbers simplifies matters if the seismic source is occasionally one of the four independent types. For a vertical strike-slip $(m=2)$, Eq. (18) can be reduced to

$$
f_{1}(\theta, \varphi) k_{n 2}^{12}=g(\theta, \varphi)
$$

Thereby, the dislocation Love number can be written as

$$
k_{n 2}^{12}=g(\theta, \varphi) / f_{1}(\theta, \varphi) .
$$

Similarly, we may obtain dislocation Love numbers for the other three source types as

$$
\begin{aligned}
& k_{n 1}^{32}=g(\theta, \varphi) / f_{2}(\theta, \varphi) \\
& k_{n 0}^{22}=-g(\theta, \varphi) / f_{3}(\theta, \varphi) \\
& k_{n 0}^{33}=g(\theta, \varphi) / f_{3}(\theta, \varphi) .
\end{aligned}
$$

However, in this special case, not all, but only the concerned dislocation Love numbers can be determined.

For a tensile source, similar equations exist as (18)(33), but with slightly different contents for $f_{i}(\theta, \varphi)(i=$ $1,2,3,4)$. They are omitted here because a pure tensile rupture occurs very rarely in practice. If necessary, they can be written out easily in the same manner as those for the shear source.

\section{Synthetic Test-Case of the 2004 Sumatra Earthquake (Mw 9.1)}

In this section, we perform a simulation test for the 2004 Sumatra-Andaman earthquake (Mw 9.1) in order to prove the validity of the method proposed here. The gravity potential coefficients determined by GRACE after this earthquake have not been released yet. However, our test study is very realistic, and the whole simulation procedure could be directly applied for the actual GRACE data after their release in the future.

The 2004 Sumatra-Andaman earthquake occurred about $100 \mathrm{~km}$ off the west coast of the Northern Sumatra, causing a devastating tsunami that hit coastlines across the Indian Ocean. The main geological background of the earthquake is that the relatively dense Indo-Australian plate subducts beneath the lighter Burma plate with a relative velocity of about $6 \mathrm{~cm} /$ year (Khan and Gudmundsson, 2005). On 26 December 2004, the two plates moved several meters, releasing stress accumulated over hundreds of years. Several fault models have been proposed for this earthquake (e.g., Ammon et al., 2005). The parameters used in the calculation are listed in Table 1 (Yamanaka, 2004). Note that these parameters were obtained solely from seismological data, and may underestimate the whole moment released by this earthquake. However, the above theory and the following numerical results show that the parameters change the seismic factor $g_{0} U d S / a^{2}$ somehow but do not affect the inversed dislocation Love numbers for the current simulation. For the actual GRACE data, a more realistic fault model should be considered.
Table 1. Source parameters of the 2004 Sumatra earthquake.

\begin{tabular}{cc}
\hline Parameter & Fault \\
\hline Strike angle & $340^{\circ}$ \\
Dip angle & $8^{\circ}$ \\
Slip angle & $112^{\circ}$ \\
$L \times W \times U$ & $1135 \mathrm{~km}^{3}$ \\
Latitude & $3.251^{\circ} \mathrm{N}$ \\
Longitude & $95.799^{\circ} \mathrm{E}$ \\
Depth & $10 \mathrm{~km}$ \\
\hline
\end{tabular}

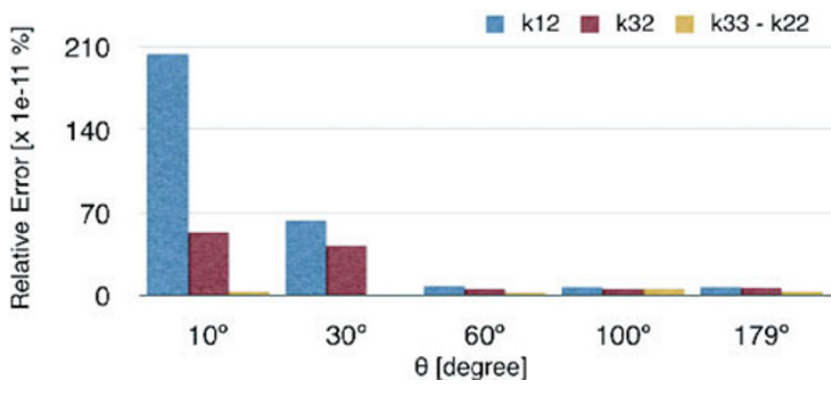

Fig. 1. Relative errors of the inverted dislocation Love numbers for degree $n=2$, based on various discretization cap area, i.e. $10^{\circ}-179^{\circ}$ in the epicentral distance.

At first, we assume no errors exist in GRACE observation. It means that the difference $\left(\Delta C_{n m}, \Delta S_{n m}\right)$ in (12) and (13) of two sets of geo-potential models $\left(C_{n m}^{1}, S_{n m}^{1}\right)$ and $\left(C_{n m}^{2}, S_{n m}^{2}\right)$ observed by GRACE contains the pure coseismic deformation. On the other hand, synthetic data can be created by known dislocation Love numbers, e.g., for degree $n=2$

$$
\begin{aligned}
& k_{22}^{12}=0.00437720437 \\
& k_{21}^{32}=0.0002946987178 \\
& k_{20}^{33}-k_{20}^{22}=-0.0268207390418 .
\end{aligned}
$$

Next, we calculated the function $g(\theta, \varphi)$ using (23), taking the coordinate origin at the epicenter of the earthquake. We then discretize the functions $f_{i}(\theta, \varphi)(i=1,2,3)$ and $g(\theta, \varphi)$ in $(19)-(23)$ by $1^{\circ} \times 1^{\circ}$, so that the elements in $\mathbf{G}, \mathbf{F}$ can be obtained. After the discretized linear equation (24) is prepared, the dislocation Love numbers vector $\mathbf{K}$ can be solved by (28). To investigate the convergence of the solutions, we first solve the linear equations by considering a cap around the epicenter with radius (epicentral distance) of $10^{\circ}$, with a system of 3600 equations. The inversed dislocation Love numbers are listed in Table 2 (the second row). It shows that dislocation Love numbers are obtained with an error less than $2.49^{-9}$ relative to the true values. Better results are obtained by increasing the radius gradually to the whole earth (Table 2) while the relative error decreases correspondingly (see Fig. 1).

Similarly, the inverted dislocation Love numbers for degrees $n=2-100$ are calculated. The relative errors are plotted in Fig. 2. It is shown that the relative errors for all degrees are less than $10^{-12}$. It indicates than the method 
Table 2. Theoretical dislocation Love numbers and the inverted results for degree $n=2$, obtained for various discretization cap area with the epicentral distance from $10^{\circ}$ to $179^{\circ}$. The numbers in the brackets give the error relative to the theoretical value.

\begin{tabular}{cccc}
\hline & $k_{22}^{12}$ & $k_{31}^{32}$ & $k_{20}^{33}-k_{20}^{22}$ \\
\hline Theoretical value & 0.00437720437000000 & 0.00029469871780000 & -0.02682073904180000 \\
$\theta=1^{\circ}-10^{\circ}$ & 0.00437720436991047 & 0.00029469871779842 & -0.02682073904179054 \\
$\theta=1^{\circ}-30^{\circ}$ & $(2.04 e-09)$ & $(5.35 e-10)$ & $(3.52 e-11)$ \\
& 0.00437720437002778 & 0.00029469871779875 & -0.02682073904180265 \\
$\theta=1^{\circ}-60^{\circ}$ & $(6.34 e-10)$ & $(4.23 e-10)$ & $(9.88 e-12)$ \\
& 0.00437720437000351 & 0.00029469871779984 & -0.02682073904180550 \\
$\theta=1^{\circ}-100^{\circ}$ & $(8.01 e-11)$ & $(5.27 e-11)$ & $(2.04 e-11)$ \\
& 0.00437720437000321 & 0.00029469871779984 & -0.02682073904181473 \\
$\theta=1^{\circ}-179^{\circ}$ & $(7.32 e-11)$ & $(5.40 e-11)$ & $(5.49 e-11)$ \\
& 0.00437720437000325 & 0.00029469871779983 & -0.02682073904180870 \\
\hline
\end{tabular}

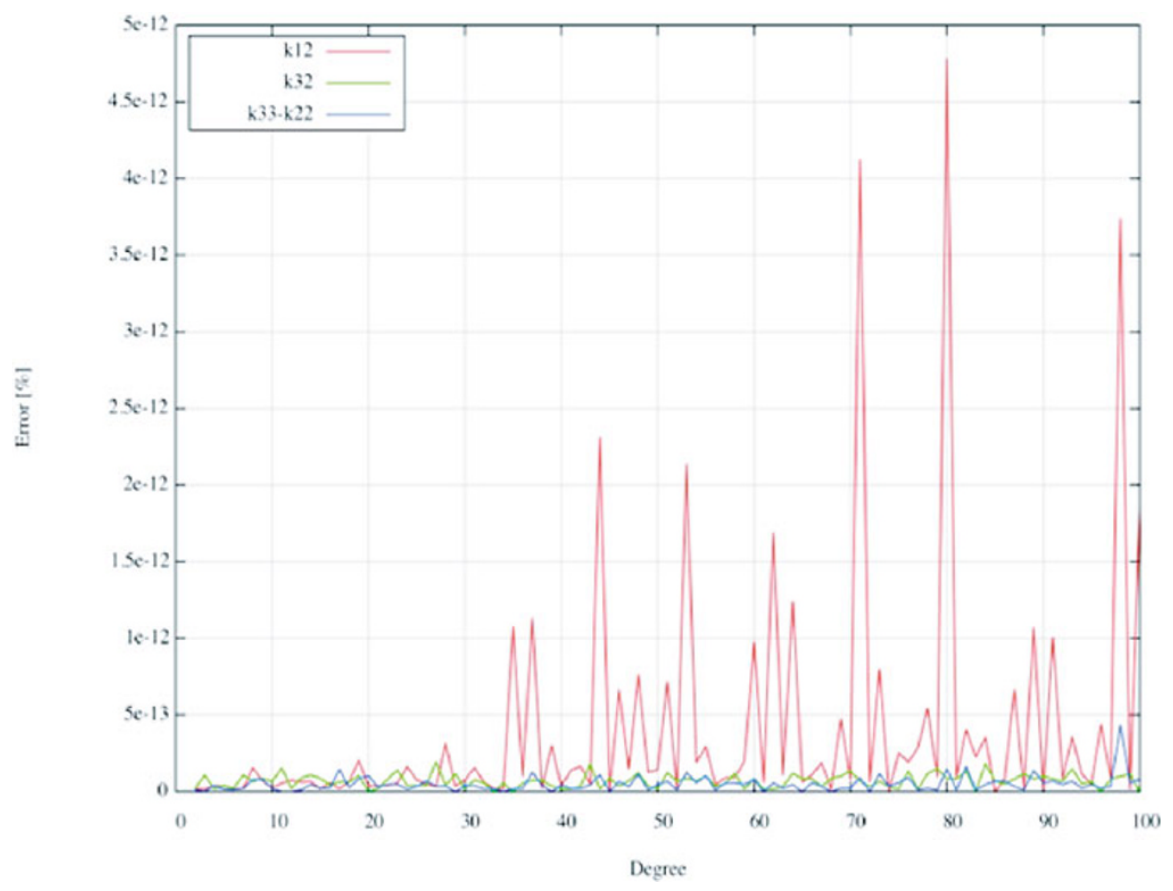

Fig. 2. The relative error of the inversed dislocation Love numbers for degree $n=2-100$.

proposed here works well for inversing dislocation Love numbers from a satellite gravity mission, provided the observation is accurate enough. It should be pointed out that relatively large errors for the parameters $k_{n 2}^{12}$ in Fig. 2 are considered to be due to the original input of the dislocation Love numbers (see (34)). That is, $k_{n 2}^{12}$ has less valid digits (nine digits) in comparison with the other two (10 and 12 digits for $k_{n 1}^{32}$ and $k_{n 0}^{33}-k_{n 0}^{22}$, respectively).

We further check the method by assuming a $1 \%$ random error in the GRACE data for degree $n=100$. The corresponding results are listed in Table 3. It shows that the estimated dislocation Love numbers are as accurate as the GRACE data (1\%) (Fig. 3). It implies that if an earthquake is large enough to cause gravity signature detectable by a gravity satellite mission, the dislocation Love numbers can be obtained by this method with sufficient accuracy. It should be pointed out that the $1 \%$ random error used here is an assumption. The purpose of the investigation is to observe whether or not the method guarantees the same accuracy as the GRACE observation. Since the real GRACE data for high degrees get less accurate as $n$ increases, there should be a certain limit to the determinable maximum degree/order of the dislocation Love numbers. However, the above results show that only if the coseismic signal is larger than the accuracy for certain degree $n$, the corresponding dislocation Love numbers can be determined.

\section{Discussion and Final Remarks}

This study presents a new method to calculate dislocation Love numbers using observations of a satellite gravity mission such as GRACE. A necessary condition is that the co-seismic deformations (e.g., potential change) should be 
Table 3. Estimated dislocation Love numbers of $n=100$ for the discretization cap area with the epicentral distance $10^{\circ}, 30^{\circ}$ and $60^{\circ}$. The geo-potential model by GRACE is considered to be more accurate than the co-seismic signals by two orders of magnitude. The numbers in the brackets give the errors relative to the theoretical value.

\begin{tabular}{cccc}
\hline & $k_{22}^{12}$ & $k_{31}^{32}$ & $k_{20}^{33}-k_{20}^{22}$ \\
\hline Theoretical value & $1.66875077200000 \mathrm{e}-04$ & $3.28713649800000 \mathrm{e}-04$ & $9.50572394700000 \mathrm{e}-04$ \\
$\theta=10^{\circ}$ & $1.67996570262265 e-04$ & $3.30348613882692 e-04$ & $9.55049795206746 e-04$ \\
& $(0.67 \%)$ & $(0.49 \%)$ & $(0.47 \%)$ \\
$\theta=30^{\circ}$ & $1.67733609659271 e-04$ & $3.30324454394680 e-04$ & $9.55141835750042 e-04$ \\
& $(0.51 \%)$ & $(0.49 \%)$ & $(0.48 \%)$ \\
$\theta=60^{\circ}$ & $1.67838920786571 e-04$ & $3.30344969817822 e-04$ & $9.55151237245961 e-04$ \\
& $(0.57 \%)$ & $(0.49 \%)$ & $(0.48 \%)$ \\
\hline
\end{tabular}

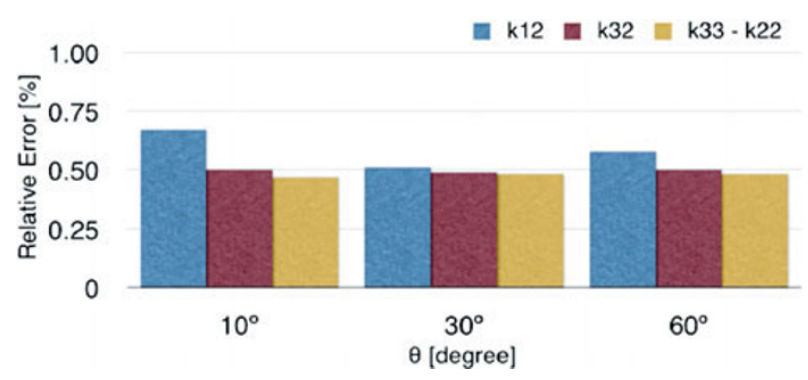

Fig. 3. The relative error of the inversed dislocation Love numbers for degree $n=100$. The geo-potential model observed by GRACE is assumed to be more accurate than the co-seismic deformation by two orders of magnitude.

sufficiently large to be detectable by the gravity mission. Deformations for each spherical harmonic degree $n$ are decoupled. Therefore, the dislocation Love numbers can be determined independently for each $n$. However, the maximum determinable harmonic degree $n$ depends on the seismic size, source type, and the accuracy (detectability) of a satellite gravity mission. For example, for a seismic event as large as the 1964 Alaska earthquake, the dislocation Love numbers of the first 70 spherical harmonic degrees are expected to be determinable using GRACE observations (Sun and Okubo, 2004a, b). Since the 2004 Sumatra earthquake (Mw 9.1) is a little bit smaller than the Alaska earthquake, it is hopeful to be detected by GRACE, but the detectable harmonic degrees should be less than 70 . The forthcoming gravity mission GRACE follow-on is expected to improve accuracy by two orders (Watkins et al., 2000). Therefore, more harmonic degrees are expected to be determined. On the other hand, for an arbitrary seismic source (with certain dip angle, e.g., around $\delta=45^{\circ}$ ), all types of dislocation Love numbers can be determined using only one seismic event because all deformation components are involved together. However, only the related dislocation Love numbers can be computed in the case of any one of the four types of sources, e.g., only $k_{n 2}^{12}$ can be obtained for a vertical strikeslip earthquake. A simulation test is carried out by considering a similar case to the Sumatra earthquake, in order to prove the validity of the method proposed in this study. Results show that the method works well and guarantee the accuracy. Since the dislocation Love numbers are expected to be sensitive to local structure of the earth, it is considered to be more reasonable to obtain them from a realistic observation such as GRACE. In addition, there are certain relation between the dislocation Love numbers and other conventional Love numbers, such as tidal Love numbers and load Love numbers (Okubo, 1993). Once the dislocation Love numbers are obtained, other dislocation Love numbers are also expected to be obtained. Since GRACE provided real observation data since about two years ago, many relative application studies have been performed, such as detections of seasonal/secular mass changes (Tapley et al., 2004; Tamissiea et al., 2005). It is also expected to apply real GRACE data to studying co-seismic deformations in the near future.

Acknowledgments. This research was supported financially by JSPS Grant-in-Aid for Scientific Research (C16540377) and "Applications of Precise Satellite Positioning for Monitoring the Earth's Environment". The authors sincerely thank Dr. R. Gross and an anonymous reviewer for their useful comments and suggestions that greatly approve the manuscript.

\section{Appendix A. Transformation coefficients $a_{n m}^{k}$ and $b_{n m}^{k}$}

According to Xu and Jiang (1964), the transformation coefficients $a_{n m}^{k}$ and $b_{n m}^{k}$ used in (15) and (16) can be derived by the following recurrence formulas, assuming $\left(\theta_{0}, \varphi_{0}\right)$ as the orientation of the seismic source, i.e.

$$
a_{n m}^{0}=P_{n m}\left(\cos \theta_{0}\right) ; \quad b_{n m}^{0}=0
$$

for $k=1$ :

$$
\begin{aligned}
(n-m+1) a_{n+1, m}^{1}= & n \cos \theta_{0} a_{n m}^{1}+\sin \theta_{0} a_{n m}^{0} \\
& -\frac{1}{2}(n-1) n \sin \theta_{0} a_{n m}^{2} \\
(n-m+1) b_{n+1, m}^{1}= & n \cos \theta_{0} b_{n m}^{1}+\sin \theta_{0} b_{n m}^{0} \\
& -\frac{1}{2}(n-1) n \sin \theta_{0} b_{n m}^{2} \\
a_{n, n}^{1}= & (n-1) \sin \theta_{0} a_{n-1, n-1}^{1} \\
& +\left(b_{n-1, n-1}^{0}-\cos \theta_{0} a_{n-1, n-1}^{0}\right) \\
& +\frac{1}{2}(n-1)(n-2)\left(b_{n-1, n-1}^{2}+\cos \theta_{0} a_{n-1, n-1}^{2}\right) \\
b_{n, n}^{1}= & (n-1) \sin \theta_{0} b_{n-1, n-1}^{1} \\
& +\left(a_{n-1, n-1}^{0}-\cos \theta_{0} b_{n-1, n-1}^{0}\right)
\end{aligned}
$$




$$
+\frac{1}{2}(n-1)(n-2)\left(a_{n-1, n-1}^{2}+\cos \theta_{0} b_{n-1, n-1}^{2}\right)
$$

for $k \geq 2$ :

$$
\begin{aligned}
(n-m+1) a_{n+1, m}^{k}= & (n-k+1) \cos \theta_{0} a_{n m}^{k} \\
& +\frac{1}{2} \sin \theta_{0} a_{n m}^{k-1} \\
& -\frac{1}{2}(n-k)(n-k+1) \sin \theta_{0} a_{n m}^{k+1} \\
(n-m+1) b_{n+1, m}^{k}= & (n-k+1) \cos \theta_{0} b_{n m}^{k} \\
& +\frac{1}{2} \sin \theta_{0} b_{n m}^{k-1} \\
& \quad-\frac{1}{2}(n-k)(n-k+1) \sin \theta_{0} b_{n m}^{k+1} \\
a_{n, n}^{k}= & (n-k) \sin \theta_{0} a_{n-1, n-1}^{k} \\
+ & \frac{1}{2}\left(b_{n-1, n-1}^{k-1}-\cos \theta_{0} a_{n-1, n-1}^{k-1}\right) \\
+ & \frac{1}{2}(n-k)(n-k-1) \\
& \times\left(b_{n-1, n-1}^{k+1}+\cos \theta_{0} a_{n-1, n-1}^{k+1}\right) \\
= & (n-k) \sin \theta_{0} b_{n-1, n-1}^{k} \\
+ & \frac{1}{2}\left(a_{n-1, n-1}^{k-1}-\cos \theta_{0} b_{n-1, n-1}^{k-1}\right) \\
+ & \frac{1}{2}(n-k)(n-k-1) \\
\times & \left(a_{n-1, n-1}^{k+1}+\cos \theta_{0} b_{n-1, n-1}^{k+1}\right)
\end{aligned}
$$

\section{References}

Ammon, C. J., J. Chen, H. Thio, et al., Rupture process of the 2004 Sumatra-Andaman Earthquake, Science, 308, 1133-1139, 2005.

Chao, B. F., Geodesy is not just for static measurements any more, Eos, Transactions, American Geophysical Union, 84, 145-156, 2003.

Chao, B. F. and R. S. Gross, Changes in the Earth's rotation and low-degree gravitational field induced by earthquakes, Geophys. J. R. Astr. Soc., 91, 569-596, 1987.

Chao, B. F., V. Dehant, R. S. Gross, R. D. Ray, D. A. Salstein, M. M. Watkins, and C. R. Wilson, Space geodesy monitors mass transports in global geophysical fluids, Eos, Transactions, American Geophysical Union, 81, 247-250, 2000.

Dziewonski, A. M. and D. L. Anderson, Preliminary Reference Earth Model, Phys. Earth Planet. Inter., 25, 297-356, 1981.

Gilbert, F. and A. M. Dziewonski, An application of normal mode theory to the retrieval of structural parameters and source mechanisms from seismic spectra, Phil. Trans. R. Soc. London A, 278, 187-269, 1975.
Gross, R. S. and B. F. Chao, The gravitational signature of earthquakes, in Gravity, Geoid, and Geodynamics 2000, edited by M. G. Sideris, pp. 205-210, IAG Symposia Vol. 123, Springer-Verlag, New York, 2001.

Heiskanen, W. H. and Z. Moritz, Physical Geodesy, Freeman, San Francisco, 1967.

Khan, S. A. and O. Gudmundsson, GPS analyses of the Sumatra-Andaman Earthquake, EOS, Transactions, American Geophysical Union, 86(9), 2005.

National Research Council, NAS, Satellite Gravity and the Geosphere, edited by J. O. Dickey, Washington, D.C., 1997.

Okubo, S., Reciprocity theorem to compute the static deformation due to a point dislocation buried in a spherically symmetric Earth, Geophys. J. Int., 115, 921-928, 1993.

Okubo, S., W. Sun, T. Yoshino, T. Kondo, J. Amagai, H. Kiuchi, Y. Koyama, R. Ichikawa, and M. Sekido, Far-Field Deformation due to Volcanic Activity and Earthquake Swarm, Vistas for Geodesy in the New Millennium, edited by J. Adam and K. P. Schwarz, International Association of Geodesy Symposia, Volume 125, 518-522, 2002.

Sun, W. and S. Okubo, Surface potential and gravity changes due to internal dislocations in a spherical earth-I. Theory for a point dislocation, Geophys. J. Int., 114, 569-592, 1993.

Sun, W. and S. Okubo, Co-seismic Deformations Detectable by Satellite Gravity Missions-a Case Study of Alaska $(1964,2002)$ and Hokkaido (2003) Earthquakes in the Spectral Domain, J. Geophys. Res., 109(B4), B04405, doi:10.1029/2003JB002554, 2004a.

Sun, W. and S. Okubo, Truncated Co-seismic Geoid and Gravity Changes in the Domain of Spherical Harmonic Degree, Earth Planets Space, 56, 881-892, 2004b.

Tamisiea, M. E., E. W. Leuliette, J. L. Davis, and J. X. Mitrovica, Constraining hydrological and cryospheric mass flux in southeastern Alaska using space-based gravity measurements, Geophys. Res. Lett., 32(20), L20501, 10.1029/2005GL023961.

Tapley, B. D., S. Bettadpur, J. C. Ries, P. F. Thompson, and M. M. Watkins, GRACE Measurements of Mass Variability in the Earth System, Science, 305(5683), 503-505, DOI:10.1126/science.1099192, 2004.

Wahr, J., M. Molenaar, and F. Bryan, Time variability of the Earth's gravity field: Hydrological and oceanic effects and their possible detection using GRACE, J. Geophys. Res., 103, 30205-30230, 1998.

Watkins, M. M., W. M. Folkner, B. F. Chao, and B. D. Tapley, The NASA EX-5 Mission: A laser interferometer follow-on to GRACE, IAG Symp. GGG2000, Banff, July, 2000.

$\mathrm{Xu}, \mathrm{H}$. and F. Jiang, Transformation of spherical harmonic expressions of gravity anomaly, ACTA Geodetica et Cartographica Sinica, 7, 252-260, 1964.

Yamanaka, Y., Off west coast of northern Sumatra, Earthquake information centre seismological note No. 161, Earthquake Res. Instit., Univ. Tokyo. (http://www.eri.u-tokyo.ac.jp/sanchu/Seis.Note/2004/EIC161e.html), 2004.

W. Sun (e-mail: sunw@eri.u-tokyo.ac.jp), S. Okubo, and T. Sugano 